\title{
Mission Concepts and Operations for Asteroid Mitigation Involving Multiple Gravity Tractors
}

\author{
2011 IAA Planetary Defense Conference
}

09-12 May 2011

Bucharest, Romania

\author{
Cyrus Foster $^{(1,2)}$, Julie Bellerose ${ }^{(1,3)}$, David Mauro ${ }^{(1,2)}$, Belgacem Jaroux ${ }^{(1)}$ \\ ${ }^{(1)}$ NASA Ames Research Center \\ M/S 202-3 Moffett Field, CA 94035, USA \\ Email:cyrus.foster@nasa.gov \\ ${ }^{(2)}$ Universities Space Research Association \\ 615 National Ave, Mountain View, CA 94043, USA \\ ${ }^{(3)}$ Carnegie Mellon University, Silicon Valley \\ NASA Research Park, Bldg. 23, Moffett Field, CA 94035, USA
}

\begin{abstract}
The gravity tractor concept is a proposed method to deflect an imminent asteroid impact through gravitational tugging over a time scale of years. In this study, we present mission scenarios and operational considerations for asteroid mitigation efforts involving multiple gravity tractors. We quantify the deflection performance improvement provided by a multiple gravity tractor campaign and assess its sensitivity to staggered launches. We next explore several proximity operation strategies to accommodate multiple gravity tractors at a single asteroid including formation-flying and mechanically-docked configurations. Finally, we utilize 99942 Apophis as an illustrative example to assess the performance of a multiple gravity tractor campaign.
\end{abstract}

Keywords: Asteroid deflection, Gravity tractor, Multiple spacecraft

\section{Introduction}

The gravity tractor (GT) concept was first proposed by Lu and Love in a 2005 Nature article [1], discussing the deflection performance of a 20 -ton nuclear-electric powered tractor. Fig. 1 illustrates the operational concept of a gravity tractor, showing the standoff distance and thruster canting angle to avoid plume impingement of the asteroid. At the NASA workshop on Near-Earth Objects (NEOs) in 2006, Schweickart et al. discussed specific applications of the concept for deflecting Apophis and 2004 VD17 with a $1000 \mathrm{~kg}$ spacecraft over. Their analysis demonstrated the applicability of gravity tractors to most NEOs making close gravitational encounters, and asteroids with diameters less than $200 \mathrm{~m}$ on a direct collision course for Earth [2]. The dynamics and control of a gravity tractor spacecraft were further discussed by Wie [3], including the use of a halo orbit to accommodate multiple tractoring spacecraft, and also by Fahnestock for large spacecraft [4].

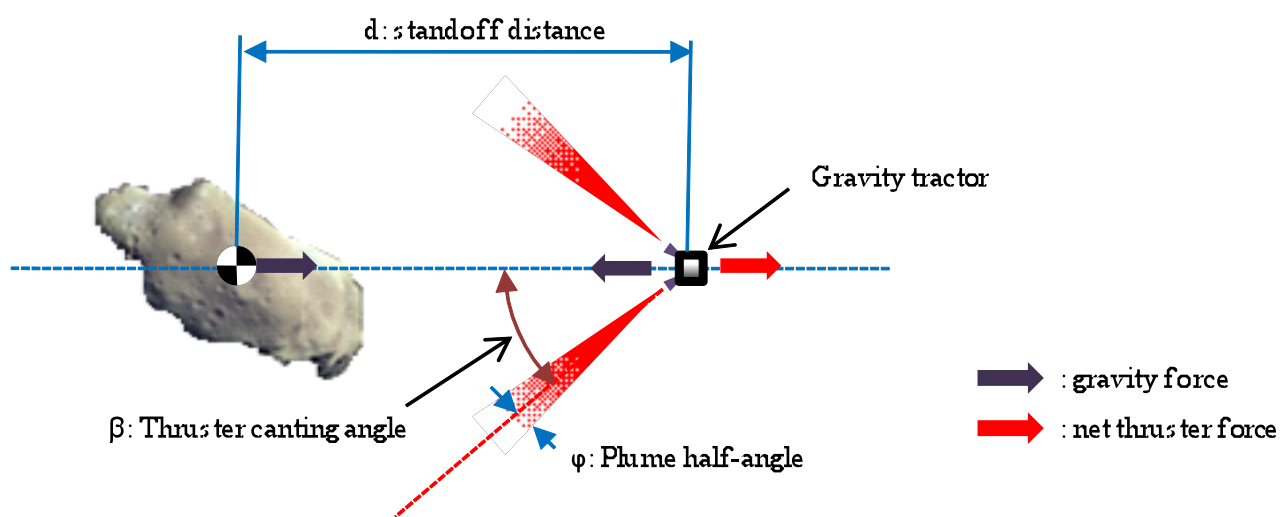

Fig. 1. Gravity tractor concept 
In 2008 JPL released a technical report on the feasibility of a 1000-kg gravity tractor preventing a gravitational keyhole entry [5]. The study assessed the improvement in orbit determination of an asteroid over ground-based observations due to the presence of a gravity tractor in the vicinity, indicating the required deflection to prevent a keyhole entry if needed. It is shown that tracking a gravity tractor in the vicinity of an asteroid for approximately a week with existing radio tracking capabilities could provide sufficient knowledge for assessing deflection requirements. Simulations were carried out to demonstrate a proposed thrust control law for the spacecraft to stay inside a bounding box maintaining a relative position from the asteroid for a stay time of up to six months. The authors also raised the geopolitical issues associated with a moving impact uncertainty region due to gravity tractor operations. A subsequent publication by the same authors and Schweickart examine the specific application of a gravity tractor deflecting 99942 Apophis from a $600 \mathrm{~m}$ gravitational keyhole in 2029 that would result in an impact at a later date, and found that such a mission to be feasible with a single 1000-kg spacecraft [6].

The use of gravity tractors is recognized in conjunction with other impact mitigation techniques such as kinetic impactors and nuclear detonations. Since a gravity tractor is required to rendezvous, it is well positioned to determine the asteroid's orbit, the necessary deflection magnitude, and feed this information to other mitigation systems. After the kinetic impact or nuclear detonation, the gravity tractor (which would have moved a safe distance away during such an event) can accurately determine the asteroid's new orbit and deliver any remaining required deflection.

\section{Multiple Gravity Tractor Missions}

Missions that include multiple gravity tractors present several advantages: increased deflection capabilities due to larger pulling mass, increased robustness against single spacecraft failure and a greater variety of mission options. A multiple gravity tractor mission can be used if the required deflection to avoid a gravitational keyhole or Earth impact exceeds the deflection capability of a single gravity tractor. In such a scenario, it will be necessary to launch more than one gravity tractor to an asteroid to deliver the required deflection.

Additionally, a small precursor spacecraft could be sent to asteroids with a non-negligible probability of entering a gravitational keyhole, followed by larger gravity tractors if a keyhole entry is confirmed. Ground observations can typically only estimate the probability of a keyhole entry or Earth impact and cannot predict them with absolute certainty. This estimate can be improved by sending a precursor spacecraft to rendezvous with the asteroid to determine its orbit more accurately through radiometry [6]. If a deflection is determined necessary, the precursor spacecraft would change roles and perform gravity tractor duties to deflect the asteroid. If however the precursor spacecraft is not massive enough to deflect the asteroid sufficiently, a subsequent larger gravity tractor, designed to provide the necessary deflection assessed by the precursor, would be launched at a later date.

Even if the deflection required to mitigate an asteroid impact is within the capability of a single gravity tractor, a multiple gravity tractor campaign would provide greater robustness against spacecraft failure. When facing a potential high-consequence impact if the mitigation mission fails, robustness and ensuring mission success is prudent. Such redundancy can be achieved through line production of several identical gravity tractors at a time, or diversity through various spacecraft contractors, independent launch vehicle providers and international partners.

\section{Deflection Performance}

We utilize an asteroid model based on 99942 Apophis for assessing the deflection performance of a multiple gravity tractor mission and comparing various proximity operation strategies. Apophis was discovered in 2004 and originally found to have up to a $2.7 \%$ probability of impacting the Earth in 2029. Subsequent observations revealed that Apophis would not intercept the Earth in 2029 but had a 1 in 135,000 chance of entering a gravitational keyhole that would result in an impact in 2036 [7]. The physical parameters used in this paper are listed in Table 1. Apophis' density and mass values are not accurately known: the assumed figures represent an upper bound from existing asteroid data yielding a conservative estimate for its mass [6].

Table 1. Assumed physical parameters for Apophis

$\begin{array}{ll}\text { Maximum radius } & 139 \mathrm{~m} \\ \text { Mass } & 6.49 \times 10^{10} \mathrm{~kg} \\ \text { Density } & 3.4 \mathrm{~g} / \mathrm{cm}^{3} \\ \text { Rotation period } & 30.4 \mathrm{hrs}\end{array}$

We assume a $1000 \mathrm{~kg}$ gravity tractor is delivered to the asteroid, of which $200 \mathrm{~kg}$ is propellant for heritage electric propulsion thrusters used on NASA's DAWN mission [6]. Other relevant spacecraft parameters are listed in Table 2. 
The gravity tractor standoff distance, as defined in Fig 1, was determined by maximizing the delivered deflection 10 years after the start of tractoring. A trade was made between the loss of thruster efficiency due to cosine losses while hovering too close to the asteroid and the weaker gravity force if hovering too far.

Table 2. Assumed gravity tractor parameters

$\begin{array}{lr}\text { Total spacecraft mass delivered to asteroid } & 1000 \mathrm{~kg} \\ \text { Propellant mass available at asteroid } & 200 \mathrm{~kg} \\ \text { Max engine thrust - each } & 90 \mathrm{mN} \\ \text { Propellant specific impulse } & 3100 \mathrm{~s} \\ \text { Exhaust plume half-angle } & 20^{\circ} \\ \text { Standoff distance to asteroid center-of-mass } & 300 \mathrm{~m} \\ \text { Thruster canting angle } & 48^{\circ} \\ \text { Exposed surface area normal to Sun } & 25 \mathrm{~m}^{2}\end{array}$

To characterize the deflection performance of a multiple gravity tractor mission, we use an analytical expression of the deflection $\Delta X$ caused by an acceleration $a_{N E O}$ as a function of the thrusting time $t_{A}$ and lead time $t_{T}$ (time from start of thrusting to deflection measurement, e.g. keyhole event or impact) [3]. This formula assumes the asteroid being deflected is in a circular orbit and that the applied acceleration is collinear with the asteroid's heliocentric velocity vector, which constitutes the optimal location for a gravity tractor to maximize deflection.

$$
\Delta X=\frac{3}{2} a_{N E O} t_{A}\left(2 t_{T}-t_{A}\right)
$$

The applied acceleration is the gravitational force between the tractor and asteroid which is proportional to the total mass of gravity tractors assuming they are located at a fixed standoff distance from the asteroid (from Newton's law of universal gravitation). We can therefore remark that the total deflection provided by multiple gravity tractors is equal to the sum of deflections provided by each gravity tractor if it were operating alone, assuming they can do so simultaneously without interfering with each other. The validity of this latter assumption will be examined in the following section.

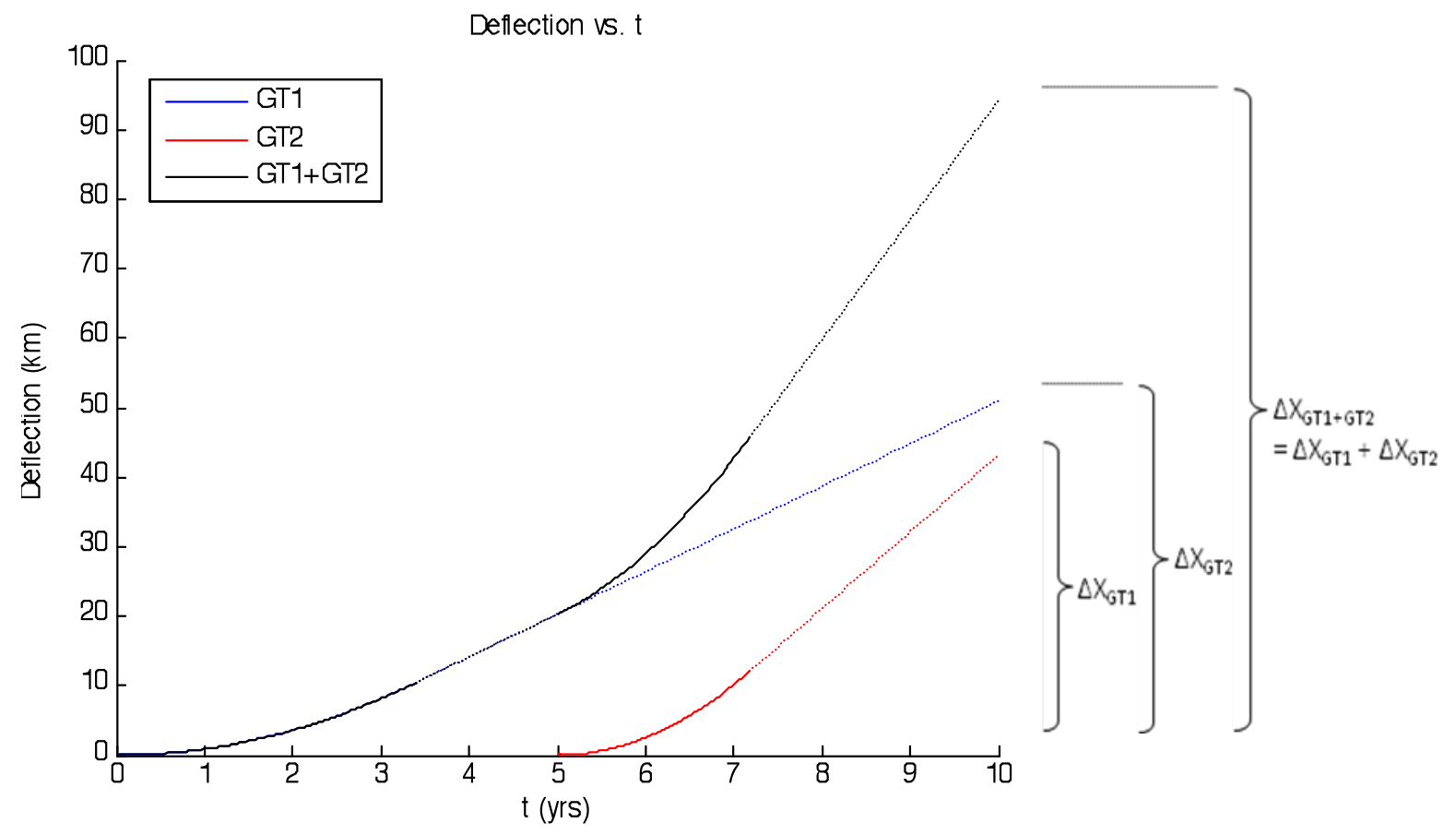

Fig. 2. Deflection of an Apophis-like asteroid resulting from two gravity tractors GT1 and GT2 acting separately and together as a function of time. GT1 and GT2 are assumed to have masses $1000 \mathrm{~kg}$ and $2000 \mathrm{~kg}$ respectively. Solid lines indicate when the GT is thrusting (a GT is modeled to continuously thrust upon rendezvous with the asteroid until fuel depletion). 
Fig. 2 illustrates the total deflection delivered by two gravity tractors calculated through numerical integration, confirming that the deflection caused by multiple gravity tractors is the sum of deflections caused by each gravity tractor if it were acting alone, even if the gravity tractors have different masses and operate at different times.

Based on these results, we can conclude that to first order, a group of gravity tractors operating simultaneously is equivalent to a single gravity tractor of equal total mass. Furthermore, based on (1), the deflection provided by a gravity tractor (or agglomeration of multiple gravity tractors) scales linearly with spacecraft mass and lead time.

\section{Proximity Operations}

If there are multiple gravity tractors operating simultaneously, their placement must be carefully considered since the optimal location of a gravity tractor of a given design is a unique point in space relative to the asteroid (along the + or velocity vector and at the optimal standoff distance previously discussed). While it is desirable to place the operating gravity tractors as close as possible to this optimal location, one must take steps to avoid any chance of collision between the spacecraft. Three options to mitigate this problem are presented in this paper: operating the gravity tractors one at the time in a serial configuration, simultaneous operation through formation-flying with strict station keeping requirements, and a parallel configuration achieved through mechanical docking of active gravity tractors. We do not seek to assess what option is best, but simply explore the operational and design requirements since such a decision would require considerations outside the scope of this paper such as spacecraft systems design, campaign planning, etc.

For each proximity operation scenario, we calculate the deflection of an Apophis-sized asteroid due to three gravity tractors simultaneously arriving 10 years before a hypothetical gravitational keyhole event. Using the asteroid and spacecraft parameters listed in Tables $1 \& 2$, we numerically integrate the path of the three gravity tractors in each configuration subject to a gravity field modeled after 433 Eros using spherical harmonics but scaled to match Apophis's size and mass [8]. Perturbations included solar radiation pressure, $3^{\text {rd }}$ body gravity from Venus, Earth, Mars and Jupiter and 1-sigma uncertainties of $1 \mathrm{~m}$ in position and $1 \mathrm{~mm} / \mathrm{s}$ in velocity relative to Apophis' center of mass. A deadband control box is assumed around the nominal tractoring point with $+/-10 \mathrm{~m}$ tolerance and maximum velocity of $5 \mathrm{~mm} / \mathrm{s}$. We consider a thruster configuration to allow translational movement along any spacecraft axis but neglect attitude control requirements (assumed to require little propellant next to translational maneuvers).

\subsection{Case 1: Serial Configuration}

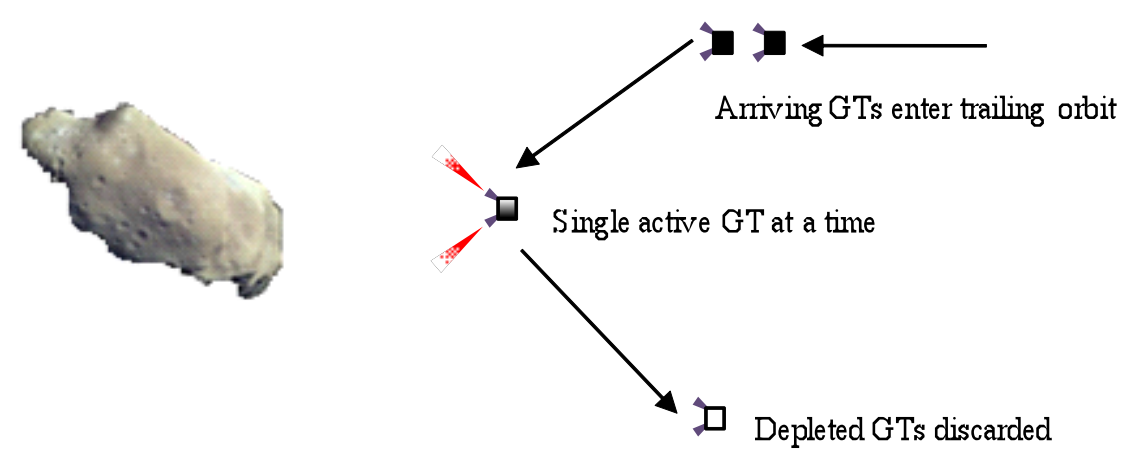

Fig. 3. Multiple gravity tractors in serial configuration

We first investigate a conservative proximity operations strategy of only operating a single gravity tractor at a time. Any backlog of gravity tractors are placed in a low-maintenance trailing orbit to await their turn at tractoring, where they are well positioned to perform radiometric tracking of the asteroid to accurately determine its orbit and the deflection imparted by the active gravity tractor. This configuration would also be used in a scenario where a single gravity tractor can deliver the necessary deflection but redundant spacecraft are placed in a trailing orbit.

In the numerical simulation, although three gravity tractors are assumed to simultaneously arrive at the asteroid 10 years before a gravitational keyhole event, they must each wait their turn for tractoring. We find that the three gravity tractors provide a total deflection of $93.8 \mathrm{~km}$ over a cumulative span of 9.64 years when operating serially until each spacecraft depletes its propellant tank. The trajectory traced by the first gravity tractor is displayed in Fig 4 during its initial month of operation. While this serial configuration is the simplest operational concept of a multiple gravity tractor campaign, we note that it provides the poorest deflection performance of the three concepts studied in this paper. 


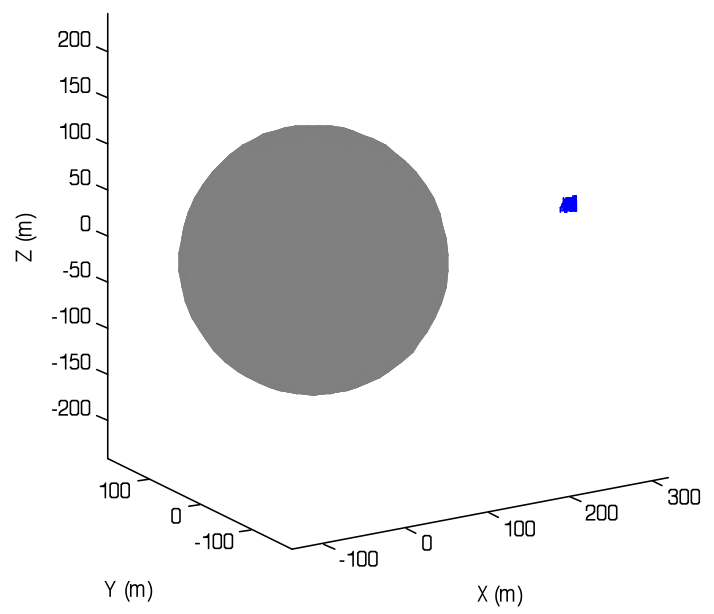

(a)

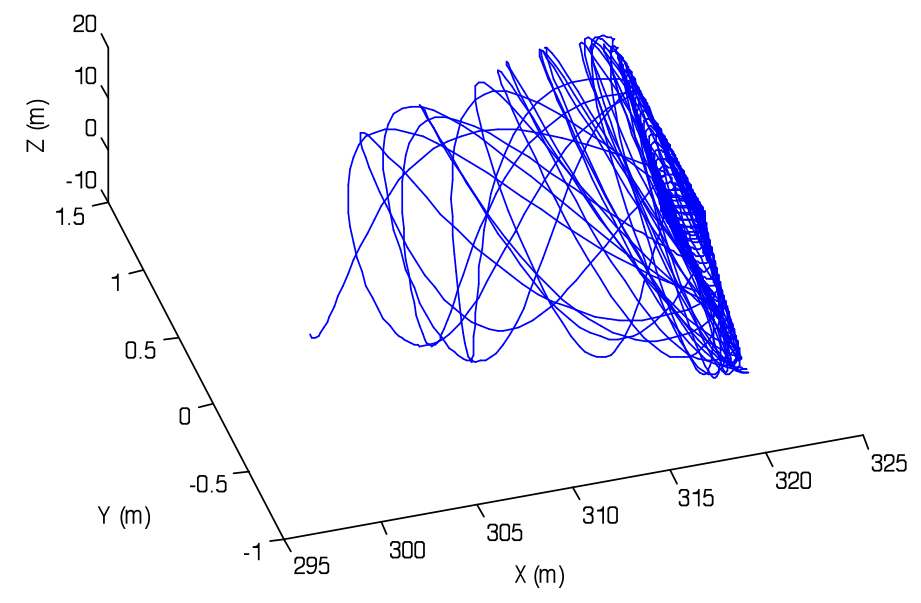

(b)

Fig. 4. Path traced by a single gravity tractor during one month of station keeping operations in a Sun-asteroid rotating frame where the optimal tractoring location is a fixed point. (a) Trajectory including Apophis, represented by a sphere but modeled with harmonics for determining gravity perturbations. (b) Close-up of trajectory.

\subsection{Case 2: Parallel, Formation-flying Configuration}
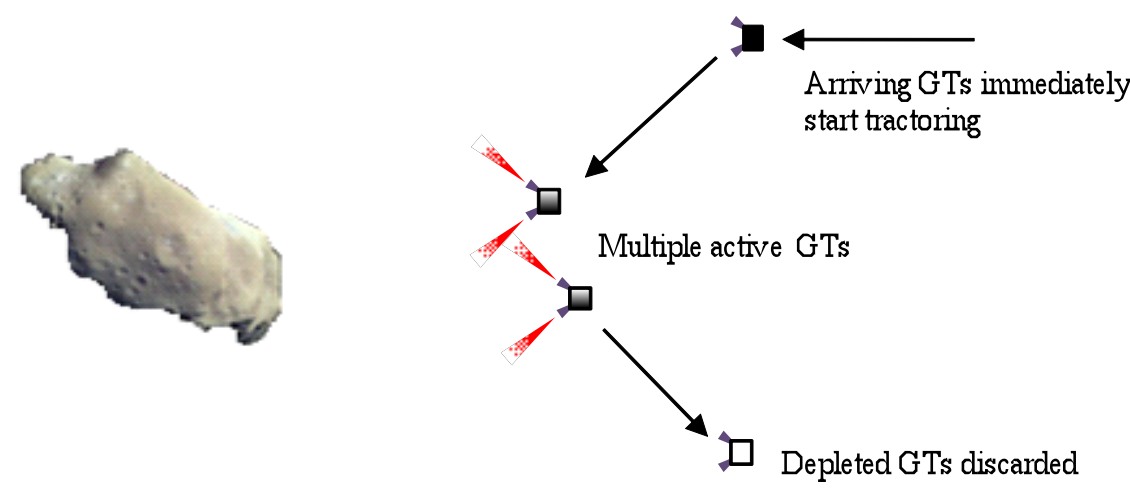

Fig. 4. Multiple gravity tractors in parallel, formation-flying configuration

We next explore a proximity operations configuration that enables simultaneous use of gravity tractors to maximize deflection since as noted from (1), it is desirable to begin tractoring operations as soon as possible after delivery to the asteroid. Active gravity tractors will have to maintain strict station-keeping requirements to not only avoid a collision but prevent plume impingement, not shade other spacecrafts' solar panels or block communications antennae. Gravity tractors designed to operate in such a configuration will likely need to be equipped with short-range radios and/or proximity cameras.

To accommodate three gravity tractors about the optimal standoff distance without risk of plume impingement, control boxes for the deadband position controller were separated by $25 \mathrm{~m}$ along a diagonal facing the asteroid as illustrated in fig. 5. Only a functional configuration was sought: the size and location of the control boxes were not optimized to maximize deflection. We note that reducing the size of the control boxes increases station keeping requirements but allows the spacecraft to be located closer to the optimal tractoring location. We find that the three-gravity tractor station-keeping configuration yields a total deflection of $149.1 \mathrm{~km}$ after 3.14 years of operations, which is as expected takes roughly $1 / 3$ as long as the serial configuration. A plot of the gravity tractors' trajectories during the initial month of operation is displayed in Fig. 6.

As an alternative to station-keeping multiple gravity tractors at a fixed location with respect to an asteroid, it is possible to insert the spacecraft into a non-keplerian halo orbit [3]. Such a configuration reduces station keeping requirements and reduces or eliminates thruster canting requirements but necessitates a more massive spacecraft to deliver the same deflection due to is offset location from the optimal tractoring location [3]. 


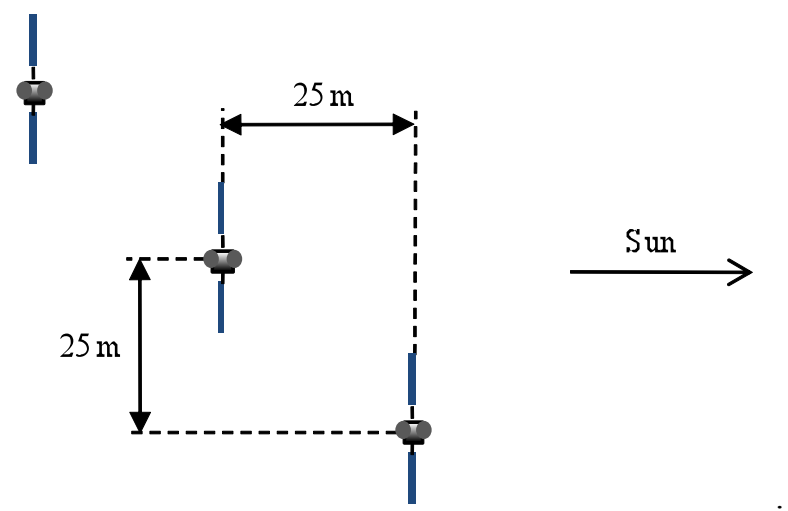

Fig. 5. Proposed positioning of three simultaneously operating gravity tractors, as viewed from the asteroid looking along its heliocentric velocity vector. The spacecraft are spaced $25 \mathrm{~m}$ apart along a diagonal in a plane facing the asteroid. Such a configuration prevents thruster plume impingement and solar panel shading.

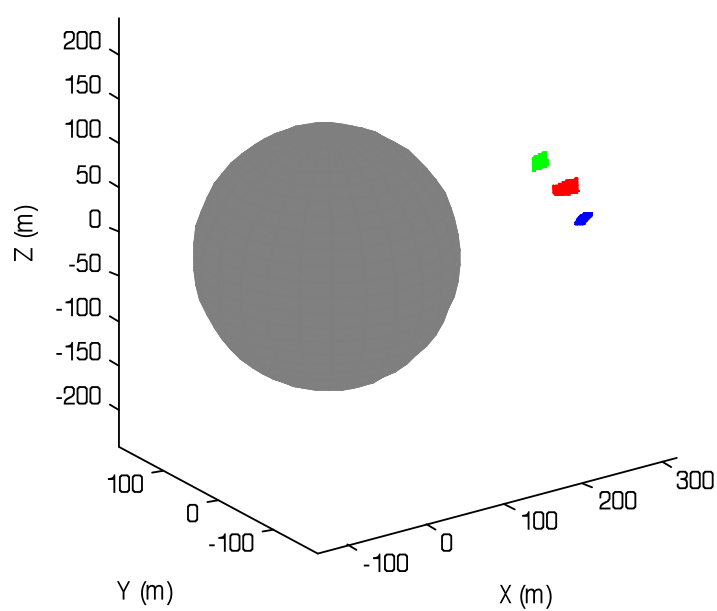

(a)

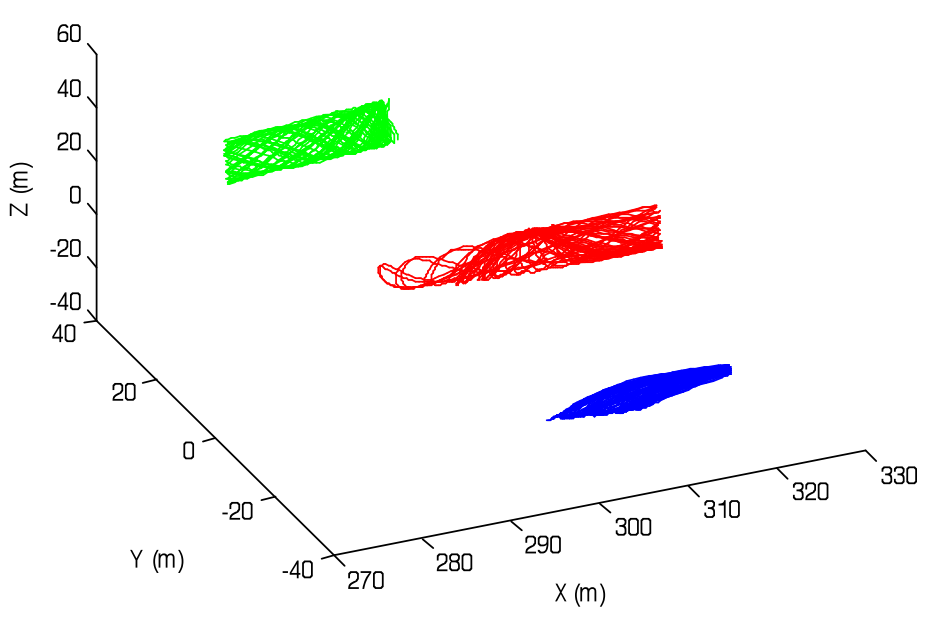

(b)

Fig. 6. Path traced by three simultaneously-operating gravity tractors during one month of station keeping operations in a Sun-asteroid rotating frame where the optimal tractoring location is a fixed point. (a) Trajectories including Apophis (b) Close-up of trajectories.

\subsection{Case 3: Parallel, Docked Configuration}
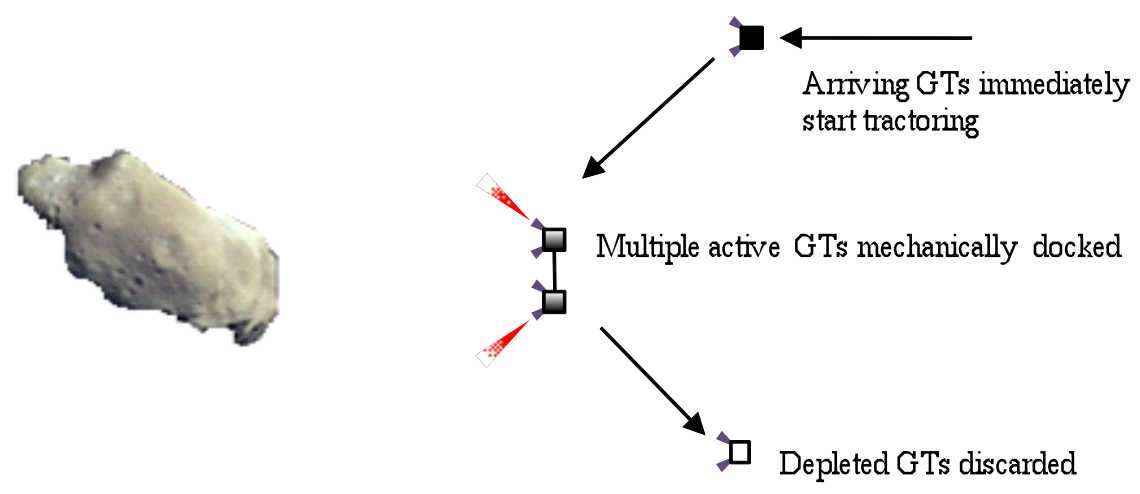

Fig. 7. Multiple gravity tractors in parallel, docked configuration

In an effort to alleviate the station-keeping requirements of case 2, we investigate a mechanical docking system that does not interfere with the gravity tractors' propulsion, allowing them to safely conglomerate at the optimal tractoring location and effectively form a single gravity tractor. The drawbacks of this configuration include the complexity of a remote docking maneuver and the design of a spacecraft to accommodate a docking interface while not shading an adjacent spacecraft's solar panels or antenna. 
A simulation of a three-gravity tractor docked configuration, assuming a $10 \mathrm{~m}$ separation distance between spacecraft center-of-masses, yields a total deflection of $155.2 \mathrm{~km}$ after 3.51 years of operations. We note a slightly greater deflection over the formation-flying configuration due to propellant saved in the less-demanding station-keeping requirements of the docked configuration. Additionally, the canting angle of the outer gravity tractors' thrusters are relaxed yielding additional tractoring efficiency since they are offset from the asteroid's centerline. The trajectory traced by the docked gravity tractors is similar in nature to the single-spacecraft case displayed in Fig. 4. A summary of simulation results for the three proximity operations configurations are presented in Table 3 .

Table 3. Deflection comparison of proximity operations configurations

Case 1: Serial

Configuration

Case 2: Parallel, formation-flying

Case 3: Parallel, docked

Deflection after $10 \mathrm{yrs}$
$93.8 \mathrm{~km}$
$149.1 \mathrm{~km}$
$155.2 \mathrm{~km}$

Required thrusting time

9.64 yrs

3.14 yrs

$3.51 \mathrm{yrs}$

\subsection{Stacking of depleted gravity tractors}
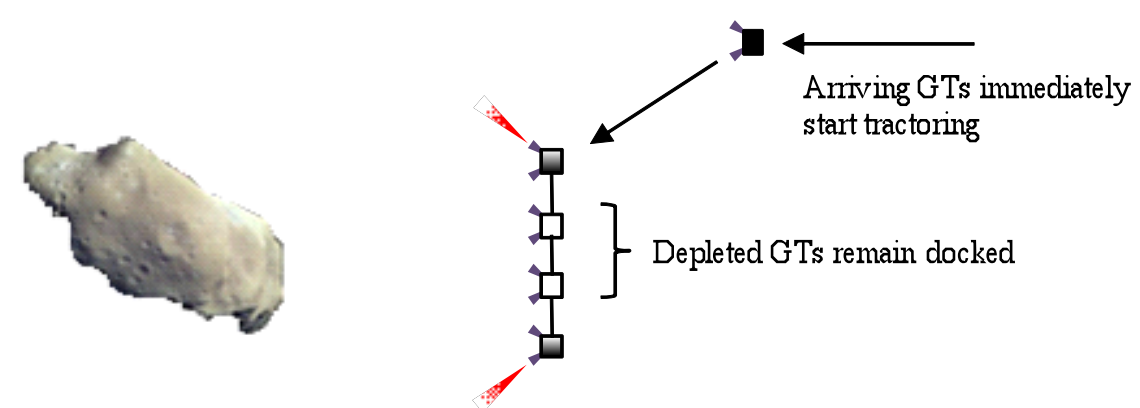

Fig. 8. Multiple gravity tractors in parallel, docked configuration with stacking of depleted spacecraft

We find that the deflection performance of the docked configuration can be improved by retaining depleted gravity tractors on the stack while active tractors remain thrusting. Fig. 9 characterizes the deflection improvement of a single active gravity tractor as a function of the number of retained docked spacecraft as calculated using (1) and with the spacecraft parameters listed in Table 2. This improvement can be explained by the extra mass of the depleted gravity tractors increasing the gravity force between the stack and the asteroid without reducing the standoff distance and hence compromising the thruster canting angle. With stacked depleted gravity tractors, the active spacecraft must consume their propellant quicker to keep up with the greater gravity force. This enhancement however cannot be taken advantage of when gravity tractors arrive simultaneously at the asteroid (as in the simulation of case 3 ) since there will never be both active and depleted gravity tractors if they begin tractor operations at the same time. Furthermore, the number of depleted spacecraft that an active tractor can accommodate is limited by its maximum thrust rating, an important limitation typical of electric propulsion systems.

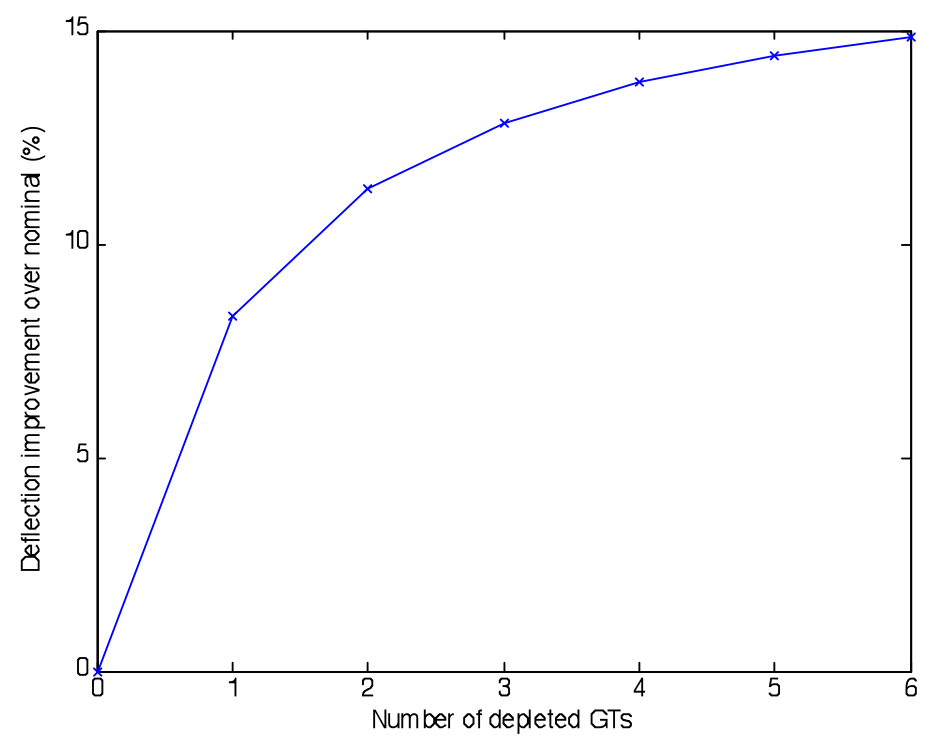

Fig. 9. Deflection improvement of a single active gravity tractor as a function of the number of retained docked spacecraft as calculated using (1) and spacecraft parameters listed in Table 2. 


\section{Campaign Example: 99942 Apophis}

We further illustrate the performance of a multiple gravity mission with a deflection campaign example to 99942 Apophis. To simulate the progressive availability of gravity tractors stemming from various spacecraft contractors and international partners, we deliver a single $1000-\mathrm{kg}$ gravity tractor at every reasonably-low $\Delta \mathrm{V}$ rendezvous opportunity with Apophis. With the six opportunities launching after 2015 and arriving no later than 2029 presented in Table 4, we seek to maximize the deflection delivered at the April $13^{\text {th }} 2029$ close-approach B-plane. One should note that although the gravitational keyhole at the 2029 B-plane that produces a resonant 2036 impact is only $600 \mathrm{~m}$ in size [6] and well within the deflection capability of a single gravity tractor, we seek in this campaign example to quantify the performance of a multiple gravity tractor mission.

In this simulation, we numerically integrate Apophis' deflected trajectory for the three proximity operations configurations discussed in the previous section. Station-keeping control is not considered which results in cases 2 and 3 (parallel, formation-flying and docked) yielding identical deflection performance. We assume that the first gravity tractor to arrive at Apophis spends 90 days tracking the asteroid before tractoring and allocate 30 days for positioning of subsequent gravity tractor arrivals.

Table 4. Rendezvous opportunities with Apophis launching after 2015 and arriving no later than April 2029

$\begin{array}{lccccc} & \text { Earth Departure } & \text { Apophis Arrival } & \begin{array}{c}\text { Time-of- } \\ \text { flight }\end{array} & \begin{array}{c}\text { Launcher } \\ \mathbf{C 3}\left(\mathbf{k m}^{2} / \mathbf{s}^{2}\right)\end{array} & \begin{array}{c}\text { Rendezvous } \\ \Delta \mathbf{V ~ ( k m / s )}\end{array} \\ \text { GT \#1 } & \text { May-15-2017 } & \text { Jun-06-2019 } & 2.06 \mathrm{yrs} & 4.7 & 2.73 \\ \text { GT \#2 } & \text { Apr-29-2020 } & \text { Jan-05-2022 } & 1.69 \mathrm{yrs} & 19.8 & 1.02 \\ \text { GT \#3 } & \text { Apr-16-2021 } & \text { Nov-21-2022 } & 1.60 \mathrm{yrs} & 31.3 & 0.19 \\ \text { GT \#4 } & \text { Jan-29-2022 } & \text { Aug-09-2022 } & 192 \text { days } & 14.5 & 2.6 \\ \text { GT \#5 } & \text { May-13-2025 } & \text { May-27-2027 } & 2.04 \mathrm{yrs} & 5.2 & 2.62 \\ \text { GT \#6 } & \text { May-16-2026 } & \text { Apr-03-2028 } & 1.88 \mathrm{yrs} & 6.9 & 2.15\end{array}$

Table 5 lists the B-plane deflection delivered for the serial and parallel configurations, along with the docked configuration employing the stacking of depleted gravity tractors which is possible in this campaign due to the staggered arrivals at the asteroid. As expected, the stacked configuration provides the greatest deflection at $134.6 \mathrm{~km}$, followed by the parallel strategies at $122.3 \mathrm{~km}$ and the serial configuration at $76.2 \mathrm{~km}$. We further confirm through numerical integration that the total B-plane deflection of a multiple gravity tractor mission is the sum of deflections caused by each gravity tractor if it were acting alone, with the caveat that depleted stacked tractors improve the performance of active ones. We observe that the deflection improvement due to stacking is most noticeable for the later gravity tractors due to the greater number of empty spacecraft. We also note that GTs \#5 and \#6 do not provide any deflection in the serial configuration - they are still in a trailing orbit waiting their turn to tractor at the April 2029 close-approach.

Table 5. Apophis deflection at April 2029 B-plane

for mission campaigns employing various proximity operations strategies

\begin{tabular}{|c|c|c|c|}
\hline \multirow[b]{2}{*}{ Deflection from } & \multicolumn{3}{|c|}{ Configuration for proximity operations } \\
\hline & Serial (case 1) & $\begin{array}{l}\text { Parallel - station-keeping } \\
\text { or docked (cases } 2 \& 3 \text { ) }\end{array}$ & $\begin{array}{l}\text { Parallel - docked with } \\
\text { stacking of depleted GTs }\end{array}$ \\
\hline GT \#1 & $39,230 \mathrm{~m}$ & $39,230 \mathrm{~m}$ & $39,291 \mathrm{~m}$ \\
\hline GT \#2 & $25,477 \mathrm{~m}$ & $28,866 \mathrm{~m}$ & $30,132 \mathrm{~m}$ \\
\hline GT \#3 & $11,220 \mathrm{~m}$ & $24,786 \mathrm{~m}$ & $26,708 \mathrm{~m}$ \\
\hline GT \#4 & $242 \mathrm{~m}$ & $25,482 \mathrm{~m}$ & $25,903 \mathrm{~m}$ \\
\hline GT \#5 & $0 \mathrm{~m}$ & $3,091 \mathrm{~m}$ & $8,346 \mathrm{~m}$ \\
\hline GT \#6 & $0 \mathrm{~m}$ & $895 \mathrm{~m}$ & $4,251 \mathrm{~m}$ \\
\hline Total & $76,169 \mathrm{~m}$ & $122,350 \mathrm{~m}$ & $134,631 \mathrm{~m}$ \\
\hline
\end{tabular}




\section{Conclusion}

We conclude that a multiple gravity tractor mission will be necessary on missions where the required deflection exceeds the capability of a single gravity tractor. We find that the total deflection scales linearly with the mass of the individual gravity tractors that make up the mission: the B-plane deflection of a multiple gravity tractor mission is the sum of deflections caused by each gravity tractor if it were acting alone, assuming they pull the asteroid along the same direction.

Several options exist for proximity operations at the asteroid. It is desirable to simultaneously operate gravity tractors upon arrival at an asteroid to maximize deflection. The mechanical docking strategy reduces station-keeping requirements and allows for the stacking of depleted tractors to improve performance. For a six-gravity tractor campaign example to Apophis with staggered launches, we find that the docked stacking strategy provides a $10 \%$ performance improvement, while that the serial configuration incurs a $38 \%$ penalty over the parallel scenarios (formation-flying or docked). The relative effectiveness of the discussed proximity operation strategies depends on many factors such as the lead time before a close-approach event, the timing of rendezvous opportunities, the asteroid mass and the gravity tractor parameters. Future work necessary to assess the practicality of a station-keeping versus docked simultaneous tractoring configuration include systems engineering of the detailed spacecraft design requirements and trading the complexity of formation flying with the intricacy of mechanical docking approach.

\section{Acknowledgements}

The authors thank NASA Ames Research Center and the Ames Mission Design Center for their support and feedback throughout this study.

\section{References}

[1] Lu, E.T., and Love, S.G., Gravitational Tractor for Towing Asteroids. Nature 438 (Nature), 2005: 177-178

[2] Schweickart, R., C. Chapman, D. Durda, and P. Hut. Threat Mitigation: The Gravity Tractor. Vail, Colorado: Submitted to NASA Workshop on Near-Earth Objects, 2006

[3] Wie, B. Dynamics and Control of Gravity Tractor Spacecraft for Asteroid Deflection. AIAA/AAS Astrodynamics Specialist Conference and Exhibit, Honolulu, Hawaii, 2008: AIAA 2008-6253

[4] Fahnestock, E.G., and D.J. Scheeres. Dynamic Characterization and Stabilization of Large Gravity-Tractor Designs, Journal of Guidance, Control, and Dynamics, 2008: 501-521.

[5] Yeomans, D.K., et al. Near-Earth Object (NEO) Analysis of Transponder Tracking and Gravity Tractor Performance. Pasadena: Jet Propulsion Laboratory, 2008.

[6] Yeomans, D.K., Bhaskaran, S., Broschart, S.B., Chesley, S.R, Chodas, P.W., Sweetser, T.H., Schweickart, R.. Deflecting a Hazardous Near-Earth Object. $1^{\text {st }}$ IAA Planetary Defense Conference: Protecting Earth from Asteroids. Granda, 2009.

[7] NASA Near Earth Object Program, Earth Impact Risk Summary for 99942 Apophis, http://neo.jpl.nasa.gov/risk/

[8] Miller, J. K., Konopliv, A. S., Antreasian, P. G., Bordi, J. J., Chesley, S., Helfrich, C. E., Owen, W. M., Wang, T. C. , Williams, B. G., D. K. Yeomans, Scheeres, D.J., Determination of Shape, Gravity, and Rotational State of Asteroid 433 Eros, Icarus 155, 3-17 (2002) 